ABSTRACT

Intermedial Ontologies: Strategies of Preparedness, Research and Design in Real Time Performance Capture The paper introduces and inspects core elements relative to the 'live' in performances that utilise real time Motion Capture (MoCap) systems and cognate/reactive virtual environments by drawing on interdisciplinary research conducted by Matthew Delbridge (University of Tasmania), and the collaborative live MoCap workshops carried out in projects DREX and VIMMA (2009-12 and 2013-14, University of Tampere). It also discusses strategies to revise manner of direction and performing, practical work processes, questions of production design and educational aspects peculiar to technological staging. Through the analysis of a series of performative experiments involving $3 \mathrm{D}$ real time virtual reality systems, projection mapping and reactive surfaces, new ways of interacting in/with performance have been identified. This poses a unique challenge to traditional approaches of learning about staging, dramaturgy, acting, dance and performance design in the academy, all of which are altered in a fundamental manner when real time virtual reality is introduced as a core element of the performative experience. Meanwhile, various analyses, descriptions and theorisations of technological performance have framed up-to-date policies on how to approach these questions more systematically. These have given rise to more sophisticated notions of preparedness of performing arts professionals, students and researchers to confront the potentials of new technologies and the forms of creativity and art they enable. The deployment of real time Motion Capture systems and co-present virtual environments in an educational setting comprise a peculiar but informative case of study for the above to be explored.

Keywords: Performance Capture, Motion Capture, The Omniscient Frame, avatar, intermediality, virtuality, performance.

BIOGRAPHIES

Matt Delbridge has a PhD from the Queensland University of Technology and lectures in Theatre Design and Performance Technologies at the Tasmanian College of the Arts. His first monograph Motion Capture in Performance: An Introduction was released in 2015, and he has also published in the journals Animation Practice, Process and Production, Body Space and Technology, and Scene. In other lives Matt has worked in orchestra management for the State Orchestra of Victoria (Aus), operated MoCap systems at the Deakin Motion Lab in Melbourne (Aus), designed for Split Britches (USA), and stage managed for Gilgul Theatre Company (Aus)

Matt.Delbridge@utas.edu.au

Riku Roihankorpi is currently the Research Director of the Centre for Practise as Research in Theatre at the University of Tampere. He has led international research projects DREX (2009-12) and VIMMA (2013-14) on technological performance. His research and teaching reflect areas of performance philosophy and intermedial, political and non-anthropocentric performance, along with their ethical challenges. Recent publications include "Performing (the Subject of) Exteriority: Virtuality, Mimèsis, and the Gratuitous 'One Must"' in Through the Virtual, Toward the Real: The Performing Subject in the Spaces of Technology (2015, Palgrave Macmillan) and the co-edited anthology Näyttämö ja tutkimus 5: Teatteri ja media(t) (2014).

riku.roihankorpi@uta.fi

\title{
Intermedial Ontologies: Strategies of Preparedness, Research and Design in Real Time Performance Capture
}

\section{MATTHEW DELBRIDGE, RIKU ROIHANKORPI}

Through analyses of performative experiments involving the real time stream of Motion Capture into modeled screen based reactive surfaces and environments, new ways of interacting in and with performances have been identified. This poses a unique challenge to traditional approaches of learning about staging, dramaturgy, acting, dance and performance design in the academy, all of which are fundamentally altered when real time virtua reality (VR) is introduced as a core element of the live performative experience. Meanwhile, various descriptions and theorizations of technological performance have framed up-to-date policies on how to approach these questions more systematically. These mentioned processes have given rise to more sophisticated notions of preparedness for performing arts professionals, students and researchers to confront the potentials of new technologies and the forms of creativity they enable. The deployment of real time Motion Capture systems and co-present virtual environments in an educational setting comprise a peculiar but informative case of study for the above to be explored.

The paper introduces and inspects core elements relative to the 'live' in performances that utilize real time Motion Capture (MoCap) or, in the present case, Performance Capture (PeCap) systems and cognate/reactive virtual reality environments. It also discusses intermedial strategies to revise manners of performing and direction, practical work processes and questions of production design, preparedness and education peculiar to technological staging. The inquiry draws on the interdisciplinary research conducted by Matthew Delbridge and the live PeCap workshops in projects Drex and Vimma between 2012-13 (University of Tampere, Finland). The project Drex developed technologies to introduce new intermedial methods of interaction to diverse spatial infrastructures, while Vimma has concentrated on the strategic development of mixed reality, virtual and sensor-based performance in live productions. Both have provided a platform for revising user-centered (performer and performance designer-centered) working methods of 1) live and telematic (remotely modified) artistic productions 2) live-oriented broadcasting, animation and game industry, as well as 3) related performer and designer education on under- and postgraduate levels.

The projects' practice-based workshops have mainly studied the unique performative environment of PeCap. Real time performance with screen space, directly translated from physical space, has been under-theorized before now, and is best understood through practical exercises undertaken in MoCap studios. The sense of space and address constructed in the exercises reveal the techniques employed to maintain an intermedial ontology that relies significantly on the performer's awareness of a virtual potential and substrate in the very process of performing. This awareness is based on witnessing and learning from specific modes of action that bring many traditional ways of directing and acting closer to the methods of technological performance. 


\section{TERMINOLOGY}

We have selected seven key terms central to the establishment of an intermedial ontology for real time Performance Capture. Definitions of Motion Capture (MoCap), Performance Capture (PeCap), The Omniscient Frame, avatar, intermediality, virtuality and performance are drawn from a variety of sources with elements to each added where necessary. The deployment of these terms, whether borrowed redefined or introduced for the first time to the lexicon of performance, assist in grounding PeCap as a legitimate mode of practice and discourse in intermedial studies.

In their account of technology and aesthetics in contemporary performance entitled Multimedic Performance, Rosemary Klich and Edward Scheer describe MoCap systems aptly as "combinations of computer hardware and software that together enable the production of 3-D digital representations of actual recorded moving bodies". ${ }^{3}$ While Klich and Scheer discuss recorded bodies and motions here, Matthew Delbridge's work, the projects Drex and Vimma, and a large number of artistic productions utilizing MoCap emphasize the real time use of the captured data. This means investing in both the real and the virtual modes of performing simultaneously and treating their specific ontological features as organic elements of an intermedial performance. Moreover, in the present study we wish to stres the term 'Performance Capture' (all performative aspects of staging a live performance with motion sensing technology and studying its peculiarities) over 'Motion Capture' (the technological mean and concepts required to do this), as the latter does not encompass the performative mode required to successfully drive, inhabit and co-exist in the binary of physical and virtual space. The research has engaged the said technology and means of performance by looking at their real and virtual potentia as a performative whole - consisting of the real and virtual settings; the concrete venue of performance; the delivery of the texts used; the overall psychophysical performance of the actors/dancers and the new experiences of live performance achieved through the work.

Just as importantly, we wish to distinguish our orientation in using the technology from othe contemporary ways of employing MoCap/PeCap, which are often economically motivated, namely performances involved with games, animation and feature films. In his Game Developer's Dictionary, Dan Carreker defines PeCap as "[h]ighly detailed recordings of the movements that make up an actor's facial expressions to reproduce them digitally". We suggest that PeCap, first properly introduced by the Robert Zemeckis feature film production The Polar Express (2004) $)^{5}$, is much more complicated than the reproduction of facial expressions. It describes the total recording of a performance withou cuts using a MoCap system. Capturing an entire performance in one take allows for traditional framing questions and dramatic devices to be employed post-performance. This in and of itself presents revolutionary concept to the preparedness of directors and performers working with recorded media and offers limitless opportunities to the ongoing development of scene and character while on set. It eases frame selection in the generation of content and allows editing to be completed after the act, in a sense returning the methods of traditional film making to the stage. The primary distinction from traditional filmmaking arrives with the theatrical approach to performance, central to PeCap, as i allows the exploration and capture of a whole scene to be undertaken in real time unhindered by device limitations (like the frame). The freedom PeCap enables is clear of the hurdles encountered in the profilmic set up and continuity of film production. It abandons the onerous repetition required with the ongoing reset and reframing of physical environments, allowing performance to occur and its inherent theatricality to re-emerge. Also, it should be noted that many animation enthusiasts tend to look with disdain at the work of Zemeckis, claiming that PeCap diminishes the role of the animator. ${ }^{6}$ We do not seek to counter these claims, but propose that an alternative view to analyzing performance and the training and preparedness of performers, directors and scenographers can be facilitated through a practical examination of performing and designing for PeCap environments. PeCap remains mostly absent in literature and as such not covered in forma studies available in our academies and institutions. Uncovering what happens in the act of generating performance in a MoCap studio reveals that there are unique production processes at play. The theorization and firming of the practice of PeCap highlights the hybridity of this mode of production.

Many descriptions of PeCap in the film industry come close to the technical parameters of our experiments, calling it an "actor-dependent and -driven" method of production whose primary instrument is the performer's imagination (with very little to work with in terms of setting etc.). They emphasize the differences and the dynamic relationships between a corporeal performance and its virtual or on-screen equivalent (for example, the need for exaggerated movements in the real). ${ }^{7}$ Yet, these approaches share the objectives of game production by relying on complex computer-driven post-production processes and by generating polished performative unitie that respond to our expectations of audiovisual storytelling and reify the modern Western interdependence between aestheticism ${ }^{8}$ and economic profit o consumption. As a partial but substantial departure from this relationship, the discussed workshops have embraced PeCap with the capricious qualities of live production, and thus with the performative capacity of glitches, kinaesthetic disproportions and aesthetic transformations occurring bic et nunc. This does not deny the fact that live PeCap experiments require close pre-production collaboration with studio technicians and specialists of coding.

Next, we will proceed to define the key concepts that comprise our understanding of the relationship between PeCap and artistic performance. First, there is the Omniscient Frame, ${ }^{9}$ a term used to describe the frame that an Optical Motion Capture system records within. The concept is intimately linked to the concept of Capture Volume, the amount of space that a MoCap system can 'see'. This revitalized frame is enabled by the capacity of a MoCap system's camera array to record within a volume, to plot and reproduce the movement of performance in a screen based impression that includes the height and width of the 2D frame of traditional film, and the captured depth of movement in space. The Omniscient Frame is the primary mechanism employed in the capture of live performance for filmic, game and theatrical production using a MoCap system. The concept of a framing mechanism that captures performance globally challenges the played intention of performance to a frame. When performance is captured in this environment, depending on the Capture Volume, all framing decisions can be mad both after and during the capture event. The Omniscient Frame challenges our understanding of performance generally as it drastically shifts the (potential) location of the audience, and reclaiming PeCap as a medium or a mode of intermediality (a method and an environment of intermedial performance) is inextricably linked to this understanding.

To provide some context around framing in MoCap it is useful to employ the more commonly understood concept of the frame from film. The neutrality of a chosen pictorial frame (as manipulated by early manual camera focus), a particula capture frame rate (as manipulated by early manual camera handles) or even capture time available (as dictated by the physical footage of film able to be carried in the camera) is only brought into question in MoCap after the movement has been captured. The truly impassive document that records a performance assumes a frame, focus and length of record that remains impartial. The standard frame capture rate (or frequency) deployed in film - the rate at which individual frames or images are captured consecutively - is 24-30 frames per second (fps). An individual MoCap Camera deployed as part of the system (that may involve up to 100 individual cameras) in a dedicated network or array captures at a standard rate of $250 \mathrm{fps}$ and contextualizes how much performance is captured in the process. This is especially the case when the frame is not limited by the restriction of the window. ${ }^{10}$

As the MoCap system and its capture of the movement of a group of markers replicates the movement of a subject in a screen based version of three dimensions, it presents a paradigm shift fo our traditional conception of the frame. Apart from a direct concentration on the actual scene, the specified performed intention is negated. Figure 1 provides a visual representation of the outer limitation of the Omniscient Frame. The deployment of a panel sphere to this renewed notion of framing still allows for the existence of the window frame that looks into the sphere facilitated by the 'panels' that make up its composition. The panel sphere offers a 
perspective of the framing MoCap enables - only limited by the size and scope of the Capture Volume the performance takes place within.

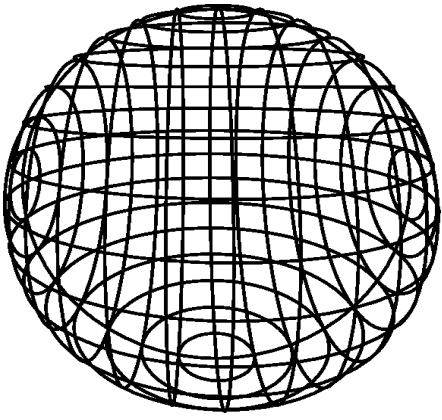

Fig. 1. The Omniscient Frame represented through Finston's Panel Type Sphere.

The existence of an 'avatar' to be activated with the captured human movement of performers is a central aspect of this work. In this instance we define the avatar not so much as Merleau-Ponty's "diabolical contraption" ${ }^{11}$, for there still resides elements of corporeality in the driving and control of this digital figure. Our notion of avatar is more akin to Craig's "Übermarionette" or Baudrillard's "clockwork counterfeit of man", ${ }^{12}$ as the presence of its driver (the performer) is a central aspect of the device's existence. The interaction(s) we explore with the avatar are in line with Sita Popat's notion of first/third person interactions where "in first-person perspective [...] effectively the screen functions as the avatar's eyes. In third-person, the [...] point of perspective floats somewhere behind (and often slightly above) the avatar, so that the avatar's body is in the centre foreground of the frame of vision."

Both the first-person and the third-person perspectives - the avatar's field of vision and a more directorial position - acknowledge the co-existence of actual and virtual objects and elements in the binary of the performance environment, and thus affect the agency of the avatar, its driver, and the specific intermedial modality/ontology they produce. These conditions can also be analyzed with Robert Wechsler' imperatives "input", "output", and "compliance", where the avatar awaits the external input/outpu condition, but remains completely connected to the performer at the centre of the MoCap environment.

As the discussed environment weaves togethe physical and virtual media and makes them interact through simultaneity of actions, we discuss 'intermediality' as an animate and non-absolute ontological state with which to observe the performative status of an intermedial stage or performer. To use the key terms set by Freda Chapple and Chiel Kattenbelt, the latter exhibits a relative freedom in moving between the tangible, "the cinematic/televisual" and "the cyberspatial" body, image and space, while maintaining a peculiar conception of time through the captured and rejoined image frames of the MoCap process. ${ }^{15}$ The performer is thus treated not as an operator or a counterpart of an audiovisual or a tangible medium (image, video, sound, set ting), but as an (inter)medium in himself, an organic means of a performative entity or continuum. Ontology, in this equation, denotes the description or the presentation of the conditions immanent to the said state - whether articulated through a performance or an analytic view. At hand is then the modern view of performance, which claims that the performers not only embody the means and the gestalt of representation, but produce the potentials of the world in and by their performance. ${ }^{16}$

Due to defining PeCap as a performative whole that does not treat its virtual element merely as a layer of representation but as a way of being in the world and producing it, some further terms need to be addressed. When virtuality is understood as a performative phenomenon in itself, it remains "a potential/capacity to realize and challenge different cultural, social and artistic practices and ideologies". ${ }^{17}$ This responds to Rob Shields' vision of the virtual, according to which " $t$ the Virtual itself can be said to be a capacity to be actualized as a singular, concrete object. Actualization is performative - th Virtual itself is a multiplicity which can be actualized in different ways. If it is known by its effects, then it is known through a specific instantiation, not as a whole. It thus retains its creative character as an ontological category pertinent to discussion of change, becoming, genesis, development, emergence, autopoesis, the genetic power of codes as well as of codings themselves." 18

The virtuality of existence is thus defined both as a potential and a substrate of actuality, "the very coherence of (its) existence", and therefor as a means of becoming, "a performance of exteriority wherein an entity becomes structured, informed, manifested and compounded; modelled without the restrictions of matter or a static $[\ldots]$ form or a model operating in the background". ${ }^{19}$ The term 'existence', then, needs to be examined as a condition of "standing outside of oneself" [cf. Lat. exsistere, to come forth], a constant process of adopting an exterior position which determines one's being as/through a subjective relation with the world", a formation of presence. ${ }^{20}$ This mode of establishing presence bears directly on the considerations of presence, environment and ecology by Gabriella Giannachi, who sees that presence "is about the continuous unfolding of the subject into what is other to it", while "environment defines $[\ldots]$ what remains other to it" but is "necessary for presence to occur". By not including "the position of the subject" but by hosting "the traces left by the

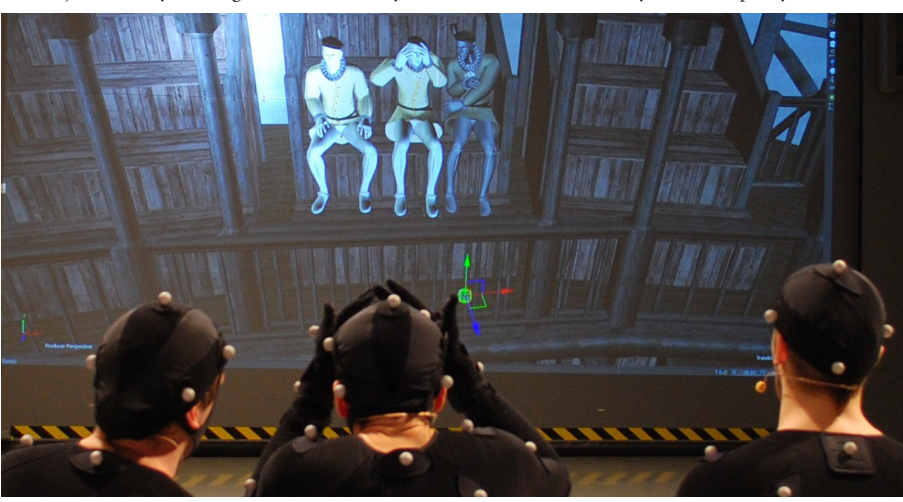

Fig. 2. Real time control of avatar(s) using MoCap system. Image: Delbridge 2012. 
and even transcend the mentioned articulations and the continuities they embody.

The above definitions provide a background for our understanding of PeCap as a mimetic capacity for forms that seek no uniform (intermedial) enunciations for conceptual approaches, but allows virtual and corporeal expressions to co-exist qua performance. The simultaneity of the latter two is central to their intermedial genealogy in informative PeCap practices. While the translation of the real to the virtual (and vice versa) does not need to refer to any distinct means of embodying ideas or intangible media, it does exhibit the co-existence of actuality and its potentials qua virtuality. The approach then communicates with what Eckersall, Grehan and Scheer mean by "dramaturgy" in their elaboration of "New Media Dramaturgy", where the former is treated as "a transformational, interstitial and translation practice" that "bridges ideas and their compositional and embodied enactment". ${ }^{22}$ However, due to the practice-led approach employed in the Drex and Vimma workshops, for dramaturgy we reserve the role of delineating the overall organization of the technological, psychophysical, textual and virtual factors of PeCap performances and the processes preparing their workflows.

TOWARDS THE INTERMEDIAL ONTOLOGIES OF PECAP: INTERACTION, PRESENCE, DIRECTION ${ }^{23}$ Matthew Delbridge's work and the projects Drex and Vimma have tackled the above questions with an international network of researchers and arts representatives. ${ }^{24}$ The practical means of approaching it have included the use of a 16 camera Motion Analysis Optical MoCap System to generate a mode of virtual and intermedial performance and the study of related virtual environments; the use of markerless MoCap systems (Kinect and Leap Motion technology); telepresent environments ${ }^{25}$ in live/ telematic productions and voice synthesis / virtual voice developed for intermedial environments. The ultimate aim of the work is their systematic use in instances of live performance, and in various broadcasting/game concepts, design/education methods and technological solutions.

The strategy has been designed to produce a field of common knowledge, which enables 1) the immediate involvement of user groups and content producers (including performers, designers, directors, PeCap supervisors and audiences) in the development processes; 2) the early involvement of the necessary acts of scaling and adapting the developed technologies and concepts in the said processes; 3) the integration of achieved results into various training modules of the arts and design education contributing to the projects and 4) the dissemination of the achieved methods, technologies, concepts and user contexts in established and prospective networks. Therefore, the analytical impact of our work on PeCap can best be described by discussing the research questions present in the Drex and Vimma workshops, which have highlighted the above problematic both on an institutional and practical level. Below, we will rephrase some of these questions and the findings connected to them in order to articulate the practical and theoretica challenges peculiar to the current developments in performing with PeCap.

Digital design with MoCap systems - such as virtual modelling of spaces and performers - provides actors, dancers, choreographers, directors, dramaturges and set designers with a convertible blueprint for a potential performance environment. It assists production teams and study groups to experiment with different spatial approaches prior to any concrete arrangements. This is also a considerable challenge, as the processes of digital modelling and planning must accommodate various perceptual issues. The interactions between virtual agents or between corporeal and virtual ones are not altogether compatible with those of merely corporeal one and, mutually, the composition and the dynamics of real life actions become increasingly problematized when used to activate elements of virtual environments.

In order to contend with these complex inter actions, Delbridge's Performance Capture exercises (designed and tested as a series that explores the complexity of the physical virtual connection) were applied to participants in the projects' workshops. The participants central to this study were acting students Olli Haataja, Jaakko Ohtonen and Aleksi Holkko from the University of Tampere.
Each performed soliloques from Hamlet, Othello and Macbeth and were captured using a standard 48 marker full body marker set that did not include the additional stream of facial or hand capture. In the series of three exercises (Single Camera Exercise, Multi Camera Exercise and Hand Frame Exercise ${ }^{26}$ the notion of the circular was amplified by the performers' navigation of, and performance within a screen-based model of the Rose Theatre. ${ }^{27}$ The Rose is a venue similar in nature to the Globe, but with a smaller stage area that amplifies the cylindrical nature of the space. The exploration of the cylindrical model enhanced the spherical nature of the 360-degree capture frame and the architectural characteristics of the venue. The participants thus faced a number of navigation challenges, and the delivery of the soliloquies - rhythm, orientation, physical emphases, spatial distances, inter-human/ avatar dynamics - not only informed the historical subtext of the performances, but also offered the actors a new intermedial mode of communicating and altering their performative presence. Initially, they found it hard to decide whether to concentrate on physical acting techniques (replicated by the screen) or to rely on the psychology of the texts, but the 360-degree frame helped each performer to observe the overall context of their actions and thus to asso-

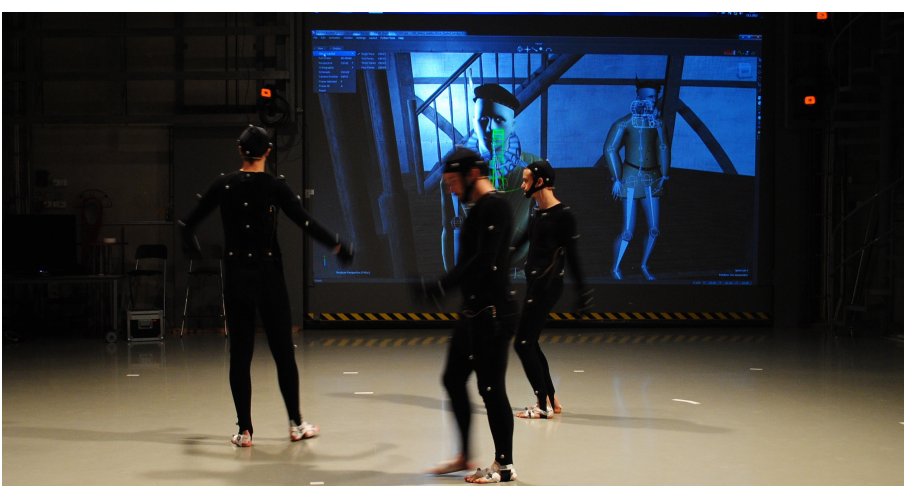

Fig. 3.Virtual cameras mounted into marker set. Image: Delbridge 2012. ate the two aspects.

Spatial feedback and simulation can be explored practically in MoCap by placing virtual cameras within the performer's marker set. Connecting aspects of replicated global framing through the real time capture and stream of performance captured, within the 360-degree camera array, facilitates the relationship between physical and virtual space. Aspects of the circular attention of space are amplified when the virtual camera is placed within the marker set. When the performer uses the virtual camera as a device, it enables focus on their performance in vironment their movement populates. This further enhances an understanding of circular space and encourages the performer to adapt perspectives of orientation. The virtual camera is then central to two modes of circular exploration. The first mode concentrates on solo performance (single camera) and the second on group performance (multiple cameras). In the foreground of Figure 3 you can see the virtual cameras revealed in the avatar (SAA $)^{28}$ on the screen. One is highlighted in green in the centre of the screen, and the other on the right hand side sitting at the top of the revealed skeleton. terms of the architectonics of the screen-based enspatial design and direction to his psychophysical 


\section{SINGLE CAMERA EXERCISE}

In this exercise a virtual camera is placed within the upper chest of the subject to generate a single perspective in virtual space. This transplants the performer from the physical environment onto (or into) the screen with a perspective aimed at replicating the 'vision' of the object. Through various incarnations of camera placement the chest emerged as the most stable camera position as it provides the least amount of movement. When the camera is placed on the head marker, for example, the environment constantly shifts. While it may seem that the camera perspective is best placed above the neck, the amount of movement generated from this position is remarkable. In Figure 3 the background screen shifts with minimal movement of the actors as they seek a mode of performance that can connect with the upper balcony of The Rose Model.

This exercise expands an ongoing understanding of the manners of directing and performing and practical work processes central to PeCap. It provides a primary connection between the physical and the virtual. The avatar with the camera becomes a medium of revealing the acute dramaturgical functions and positions of the fellow avatars and the virtual space, all of which need to be explored by acknowledging the affordances of both the concrete

and the screen space. This performative ecology that draws on and navigates the virtual - assumed, imagined and subsequently embodied - aspects of the real and the immediate output of the virtual venue and subjects on screen, corresponds to the way presence is produced in Giannachi - mainly by indicating the necessary elements of environment within individual (intermedial) performance. Each given act and reaction of the performed camera - the very production of its presence - builds up a new conception of the environment the performer occupies and entails compliance with new syntheses of the virtual and the (virtual of the) real (see Figure 4). The in termedial composition and ontology thus achieved not only informs all performers of the stances, distances and anticipations marking the situation but also teaches them the peculiar acts and kinesthetic manners of framing required to expose and focus on the virtual elements essential for their performance and the exercise's psychophysical impact.

\section{MULTI CAMERA EXERCISE}

The multi camera exercise contributes to a layered presence within the physical- virtual environment. In this exercise a virtual camera is placed within each of the performer's marker sets. This generates

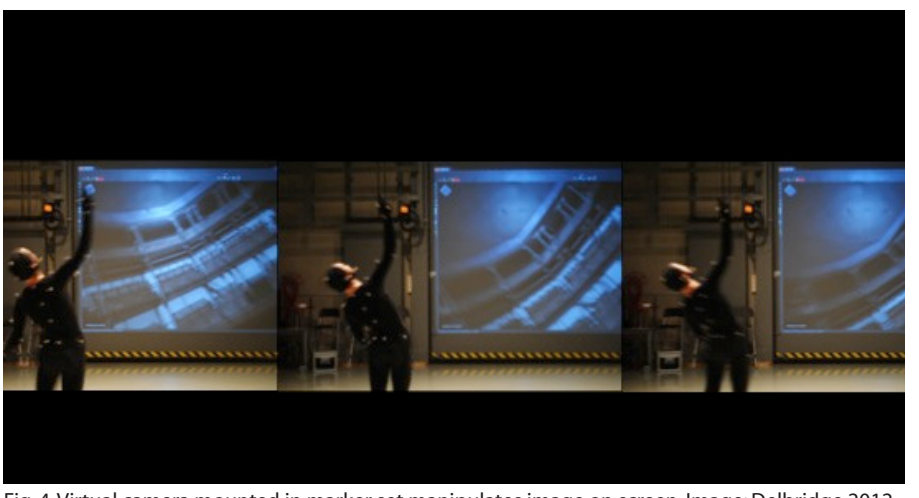

Fig. 4. Virtual camera mounted in marker set manipulates image on screen. Image: Delbridge 2012.

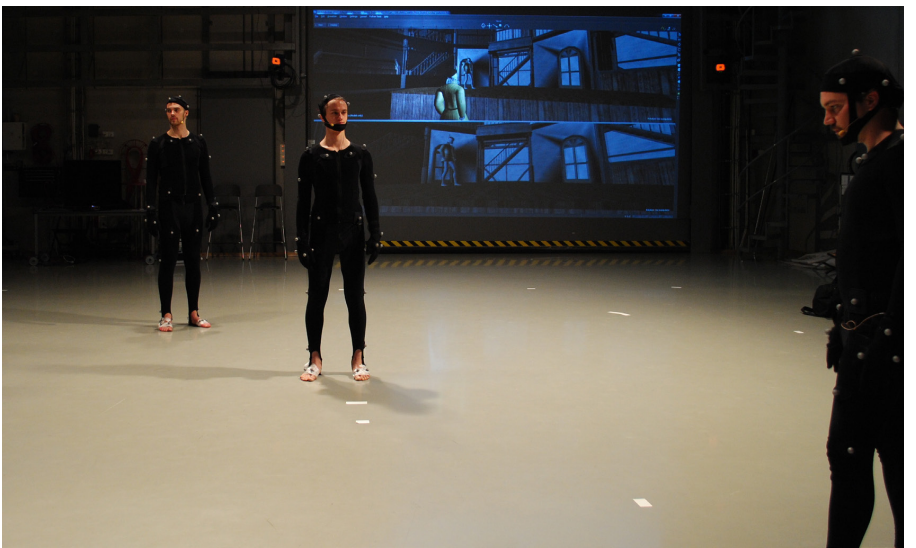

Fig. 5. Three actors placed into the environment with two mounted cameras. Image: Delbridge 2012.

the potential for the performers in the physical space to act as a virtual camera able to 'film' each other. These perspectives are replicated in the virtual environment as a visualized camera point of view (POV). In this particular act, the avatar becomes a camera and then the 'camera operator' choosing to focus on aspects of the other avatars' performance while contributing their own performance as a viewing perspective. When more than one camera is utilized, this generates a complete occupation of the screen environment and allows the director to begin to direct performers not just as performers but also as cameras. As the occupants of the virtualized environment interact with each other in real time, their streamed perspectives activate the virtualized environment in the translation of screen to physical space. In the workshop one of the performers was placed on the stage area of The Rose while the other two were placed on the ground acting as audience members for the performed scene in the theatre, and as cameras for the environment.

In Figure 5 you can see the performer in the far right standing on the same level as the other two performers (the cameras), but in screen space he occupies the position on the stage. The performer from the actor/camera even though they all perform on the same level in the studio. The multi-camera exercise demonstrates the potential for PeCap exercises to be used in the ongoing preparedness of performers in our formal training institutions. This presence enables a directorial connection, each of the performers to the other, as well as establishing a firm link to the screen where the physical properties of their actions are used to achieve the pragmatic requirements of the virtual environment. The real time occupation of interdependent forms of staging in the exercise bears directly on the psychology and "kinesthetic awareness"29 that surpasses concrete spatial arrangements and exhibits a performance's capacity to use its structure, dramaturgy and methods as an informative instance of representation to 'thoroughly furnish' the perceived outcome. The intermedial ontology peculiar to the exercise is bes described as an effective instance of adopting an exterior (virtual) motif for one's performance, since the performers were able to distinguish as many as five overlapping but interdependent levels of repin the foreground maintains a performative heigh 
resentation/operation in their performance: embodying a (performing) virtual camera, performing for a virtual audience/venue, performing for a virtua camera, performing for an actual studio environment and performing for an intermedial environment comprised of the actual and the virtual sets.

\section{CONCLUSION: EDUCATIONAL ASPECTS}

For the performers, exercising with digital technologies offers an opportunity to treat the reactive capacity of the systems as a tool for altering their own pscyhophysical orientation. Learning the functions of a MoCap suit and system, or the preparations, working methods and communicative skills required from a performer to become an organic element of the virtual machinery, serves as an introduction to several unanticipated dynamics, laws, regularities and structures. These are not altogether easy to grasp as embedding one's actions simultaneously to the physical setting and the virtual (or mixed media) environment under the terms of the physical world is an extra challenge for the performers. Movements of the body are guided by visua feedback and the bodily and spatial dimensions of virtual actants must be informed both by the varied physical means of expression and the oftentimes rigid terms of the virtual. The situation is particularly hospitable for reconsidering the directorial relation between physical acting techniques and the emotional or psychological processes comprising a performance. Anthropomorphic avatars with no distinctive features help the performers to establish a pragmatic and meaningful relationship between themselves and the intended import of their actions. Meanwhile, the performative parameters of real time MoCap and the recorded data of the use of space, trajectories of movement and the dynamics of the body allow one to take notice of unintentional mannerisms and to study the ways people (of different ages and physical traits), animals or imaginary beings move and explore their environments. The 360-degree angle of view to virtual settings also prompts the performers and the designers to observe the overall composition of a performer or a performance comprehensively. The performers of the workshops considered the mentioned elements useful not only for the PeCap experience but for their usual exercises and training methods as well. The interaction between the real and the virtual and traversing both of these sites of performance also offers new kinds of challenges for the spectators. Observing and judging the questions of naturality, genuinity or performative competence in the actions of virtual and real life performers lead to aesthetic, observational and ontological compromises and rediscoveries. In order to adapt to the virtual environment and to generate more natural movement therein, the performers sometimes need to resort to rigid and compulsive physical gestures. Yet, the plain virtual skeletons of human figures used by the MotionBuilder software to compose the virtual characters - yield peculiar experiences of species-specific recognition in the observers, even as they break and become distorted.

Despite the challenges in comprehensively grasping the virtual, intermedial and corporeal factors of a PeCap performance (and even when these challenges are considered an aesthetic or experiential function of a performance), the various mean of psychophysical interaction with virtual and media-induced entities offer the performers, designers and spectators a chance to explore different new medialities - in-between states of adaption or contrast. These states, and the intermedial ontologies they enforce - that is to say: modes, elements and logics of performing with parallel and interdependent forms of staging - generate informative experiences of what kind of embodied transformations, transgressions and dimensions a performance, performer, a content-led media production or a performer-centered media application are able to establish in the future. This concerns the strategic development of the field as well, necessitating extensive interdisciplinary work on forms of technological performance and communication between institutions operating in similar artistic contexts.

\section{NOTES AND REFERENCES}

For recent reflections on the development of performing with virtual realities and elements of mixed reality/ media, see e.g. Intermediality in Theatre and Performance, Freda Chapple and Chiel Kattenbelt, eds., Rodopi, Amsterdam and New York 2006; Chris Salter, Entangled: Technology and the Transformation of Performance, The MIT Press, Cambridge and London 2010; Mappin Intermediality in Performance, Sarah Bay-Cheng, Chic Kattenbelt, Andy Lavender and Robin Nelson, eds. Amsterdam University Press, Amsterdam 2010; Jennifer Parker-Starbuck, Cyborg Theatre: Corporeal/Technological Intersections in Multimedia Performance, Palgrave Macmillan, Basingstoke and New York 2011; Performance and Technology: Practices of Virtual Embodiment and Interactivity, Susan Broadhurst and Josephine Machon, eds., Palgrave Macmillan, Kindle ebook edition, Basingstoke and New York 2011; Rosemary Klich and Edward Scheer, Multimedia Performance, Palgrave Macmillan, Basingstoke and New York 2012.

2 The workshops were managed by the University of Tampere and Aalto University School of Arts, Design and Architecture, and took place in the Media Centre LUME (Aalto University). For further information on the projects Drex and Vimma, see: http://t7.uta.fi/drex/DREX/ IntroductionEn.html and http://www.vimmaproject. $\mathrm{com} /$

3 Klich and Scheer, op. cit., p. 111

4 Dan Carreker, Game Developer's Dictionary: A Multidis ciplinary Lexicon for Professionals and Students, Course Technology / Cengage Learning, Boston 2012, pp. 80 159.

5 Robert Zemeckis, The Polar Express (2004); see also Monster House (2006), Beowulf (2007) and A Christmas Carol (2009).

6 See Derek Hayes and Chris Webster, Acting and Performance for Animation, Focal Press, Burlington and Abingdon 2013.

7 Frank Nestor, "Performance Capture Is Acting" in Back Stage, vol. 52, no. 46, 2011, p. 12.

8 The notion of art that exists for the sake of its aesthetic and myth-induced qualities alone.

9 Delbridge first applies this term in Matthew Delbridge and Joanne Tompkins, "Reproduction, mediation, and experience: virtual reality, motion capture and early modern theatre" in Space-Event-Agency-Experience, open access e-publication of the Drex Project, Centre for Practise as Research in Theatre, University of Tampere, Tampere 2012. See also Matthew Delbridge, "The ecological approach to visual perception and the actor performance captured in the gaming landscape" in $A n$ imation Practice, Process \& Production, vol. 2, issue 1-2, 2012, pp. 137-49 and his forthcoming Palgrave Pivo title Performance Capture: An Introduction, 2015.

10 Delbridge and Tompkins, op. cit.

11 Sita Popat, "Keeping it Real: Encountering Mixed Reality in Igloo's SwanQuake: House" in Convergence: The International Journal of Research into New Media Technologies, vol. 18, no. 1, 2012, pp. 8-9.

12 Edward Gordon Craig, "The Actor and the Ubermarionette" (1907) in Craig on The Theatre, J. Michael Walton, ed., Methuen, London 1983; Jean Baudrillard, Symbo ic Exchange and Death, SAGE Publications, London, Thousand Oaks and New Delhi 1993, p. 53.

13 Popat, op. cit., p. 7 .

14 Robert Wechsler, "Artistic Considerations in the Use of Motion Tracking with Live Performers: A Practical Guide" in Performance and Technology, op. cit., pp. 65-7. 15 Chapple and Kattenbelt, "Key Issues in Intermediality in Theatre and Performance" in Intermediality in Theatre and Performance, op. cit., p. 24

16 Samuel Weber, Theatricality as Medium, Fordham University Press, New York 2004, pp. 6-8

17 Riku Roihankorpi, "Performing Exteriority: An Essay on Virtuality and Mimesis in Spatial and Agential Contexts" in Space-Event-Agency-Experience, op. cit, p. 88 . http://t7.uta.fi/drex/Drex/11_TextsAndPublicationsEn_files/7_Roihankorpi.pdf (accessed 19 February 2014).

18 Rob Shields, "Virtualities" in Theory, Culture \& Society, no. 23,2006 , p. 285 . http://tcs.sagepub.com/content/23/2-3/284 (accessed 19 February 2014).

19 Roihankorpi, op. cit.

20 Roihankorpi, op. cit., p. 92, note 14. Cf. Esa Kirkkopelto, "Näyttämön ilmiö" in Esitys katsoo meitä. Näyttämo of tutkimus 1, Pia Houni, Pentti Paavolainen, Het Reitala and Hanna Suutela, eds., Teatterintutkimuksen seura ry, Helsinki 2005, pp. 22-3.

21 Gabriella Giannachi, "Environmental presence" in $A r-$ chaeologies of Presence: Art, Performance and the Persistence of Being, Gabriella Giannachi, Nick Kaye and Michae Shanks, eds., Routledge, Abingdon and New York 2012 , p. 52

22 In Peter Eckersall, Helen Grehan and Edward Sheer 
"New Media Dramaturgy" in The Routledge Companion to Dramaturgy, Magda Romanska, ed., Routledge, London and New York 2015, pp. 375-80, manuscript.

23 Sections of this and the last two chapters will be published as part of a project description of the Vimma project (2013-14) for the Teatteri ja mediat. Näyttämö e tutkimus 5, research anthology on theatre and media of the Theatre Research Society of Finland.

24 The network includes the University of Tampere (Centre for Practise as Research in Theatre-T7), Tampere University of Applied Sciences, the Mediapolis initiative in the Pirkanmaa region, Aalto University, the University of Arts (Finland), Queensland University of Technology, the University of Queensland, Deakin University (Australia), the Royal Institute of Technology-KTH, Stockholm Academy of Dramatic Arts, the Cabaret Electrique project, Riksteatern (Sweden), the University of Salford at MediaCityUK, BBC, Manchester Metropolitan University (UK) and Trinity College Dublin (Ireland).

25 By telepresent environments we refer to physical or virtual environments reproduced in another location as well as the traversable qualities of real and virtual positions of performance.

26 Delbridge 2015, op. cit.

27 The Rose is a no longer existent Elizabethan venue. It was used as a test model for a series of workshop exercises as part of the Drex project. Modelled by Ortelia (a company based in Australia), it has many challenges that are useful in the introduction of visualized screen based spatial performance for actors.

28 The SAA is an acronym for Shakespearean Actor Avatar - the primary character used within the Drex workshop.

29 See Wechsler, op. cit., pp. 62-3. 